\title{
International Journal of Mechanical and Industrial Engineering
}

July 2013

\section{EFFECT OF EXPERIMENTAL VARIABLES ON TRIBOLOGICAL PROPERTIES OF MARTENSITIC STAINLESS STEEL}

\author{
S.G. SAPATE \\ Department of Metallurgical and Materials Engineering, VNIT, Nagpur, sgsapate@yahoo.com \\ AVISHKAR RATHOD \\ Department of Metallurgical and Materials Engineering, VNIT, Nagpur, AVISHKARRATHOD@gmail.com \\ s AHMED \\ Department of Metallurgical and Materials Engineering, VNIT, Nagpur, S.AHMED@gmail.com
}

Follow this and additional works at: https://www.interscience.in/ijmie

\author{
Recommended Citation \\ SAPATE, S.G.; RATHOD, AVISHKAR; and AHMED, s (2013) "EFFECT OF EXPERIMENTAL VARIABLES ON \\ TRIBOLOGICAL PROPERTIES OF MARTENSITIC STAINLESS STEEL," International Journal of Mechanical \\ and Industrial Engineering: Vol. 3 : Iss. 1 , Article 6. \\ DOI: 10.47893/IJMIE.2013.1121 \\ Available at: https://www.interscience.in/ijmie/vol3/iss1/6
}

This Article is brought to you for free and open access by the Interscience Journals at Interscience Research Network. It has been accepted for inclusion in International Journal of Mechanical and Industrial Engineering by an authorized editor of Interscience Research Network. For more information, please contact sritampatnaik@gmail.com. 


\title{
EFFECT OF EXPERIMENTAL VARIABLES ON TRIBOLOGICAL PROPERTIES OF MARTENSITIC STAINLESS STEEL
}

\author{
S.G.SAPATE ${ }^{1}$, AVISHKAR RATHOD ${ }^{2} \&$ S. AHMED ${ }^{3}$ \\ ${ }^{1,2 \& 3}$ Department of Metallurgical and Materials Engineering, VNIT, Nagpur - 440011, India \\ E-mail : sgsapate@yahoo.com
}

\begin{abstract}
Wear by slurry abrasion occurs during transport of abrasive slurries encountered in industrial and specially mining applications, extruders, and in slurry pumps. Steel piping systems are widely used in mining to convey the mineral, ore and tailing slurries to the processing plant, as well as to recycle the debris medium back to the mining area. Hardfacing by welding is one of the economic methods to improve wear resistance of engineering components. The present work reports slurry abrasion behaviour of hardfaced martensitic stainless steel under a wide range of experimental condition. The slurry abrasion experiments were performed using slurry abrasion test apparatus using silica sand as the abrasive medium. The effect of test parameter such as sliding distance, normal load and slurry concentration of abrasive medium on slurry abrasion behaviors of martensitic stainless steel was investigated. The slurry abrasion volume loss exhibited increasing trend with increasing sliding distance, normal load and slurry concentration, although the magnitude of increase was different in each case. The results of the investigation suggest and slurry concentration had relatively stronger effect as compared to that of normal load.. The morphological studies of the worn surfaces revealed characteristic differences in the wear pattern under different test conditions.
\end{abstract}

Keywords - stainless steel; wear; abrasion; slurry; cutting; indentation

\section{INTRODUCTION}

The primary modes of wear responsible for degradation of the engineering components in industries are sliding wear, abrasive, erosive wear and chemically assisted wear. Wear by abrasion accounts for more than $50 \%$ of the wear failures occurring in industries. The phenomenon of wear is not only responsible for material removal but also leads to premature failure of engineering components. The monetary loss due to wear also includes cost involved in replacement and downtime cost

Slurry abrasion is defined as progressive loss of material from the component in which liquid media carries abrasive particles. Wear by slurry abrasion occurs in extruders, slurry pumps, and pipes carrying slurry of minerals and ores in mineral processing industries, extruders, pump impellers and coal slurry nozzles. The wear life of components used under slurry abrasion conditions is governed by the process parameters, properties of abrasive particles in slurry and material properties. The evaluation of slurry abrasion under actual service conditions is often a difficult task, due to interactive effects of different parameters such as slurry concentration, velocity and properties of abrasive medium on wear rate. [1-4].

Steels and cast irons are widely used for improving wear life of engineering components under abrasive wear situations. The abrasive wear properties of steels have been investigated in the past. wear resistance of steels is influenced by properties of abrasive particles, test conditions and mechanical properties of steels and material properties [4,5,6-12]. Hard facing by welding is a one of the economic method to improve abrasion resistance of engineering components. A little amount of data is available on slurry abrasion behaviour of weld deposited alloy steels and in particular martensitic stainless steels. In the present work, the experiments were performed using silica sand slurry with different sliding distance, slurry concentration, and load. The morphology of the worn surfaces after slurry abrasion was studied under scanning electron microscope. The morphological studies of the worn surfaces revealed characteristic differences in the wear pattern under different test conditions.

\section{EXPERIMENTAL}

The slurry abrasion experiments were carried out on martensitic stainless steel was deposited by Manual metal arc welding process over $10 \mathrm{~mm}$ thick mild steel plate. The consumable used for deposition was in the form of electrode. The welding parameters were; Voltage $\sim 22 \mathrm{~V}$ and current $\sim 120$ A (direct current). The thickness of weld deposit was typically $4 \mathrm{~mm}$. The chemical compositions of the investigated steel are given below, determined by spark emission spectroscope was (weight $\%$ ) C- 0.0483, Mn- 0.87, Si1.07, S- 0.011, P- 0.021, Cr-15.3, Ni- 3.64, FeBalance. 


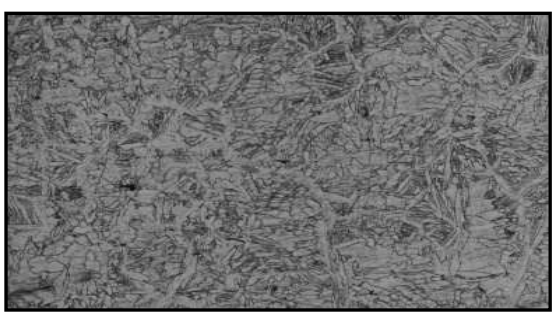

Fig 1 : Microstructure of weld deposited stainless steel showing martnesite

The specimens for hardness, metallography and abrasion testing were derived from the weld deposited plate, ground and polished with successive silicon carbide emery papers

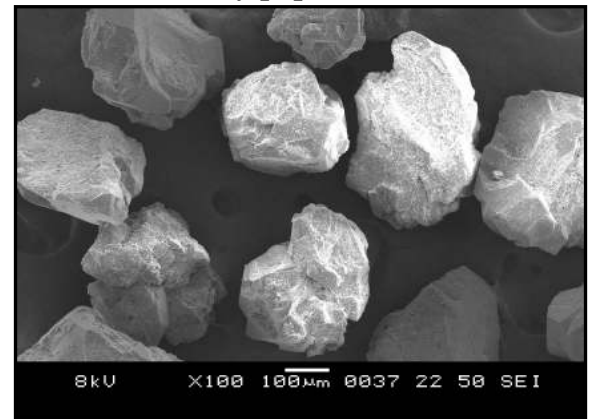

Fig. 2 : SEM photograph of silica sand particle $(250-300 \mu \mathrm{m})$

of grade $1 / 0,2 / 0,3 / 0$ and $4 / 0$, after that the specimens were subjected to alumina wheel polishing to obtain excellent surface finish. The well polished specimen of martensitic stainless steel was etched with Kalling's etching reagent. The bulk hardness measurement of martensitic stainless steel was made using Rockwell hardness tester which is $40 \mathrm{HRc}$. Silica sand was used as abrasive particle in slurry abrasion test. Abrasive particle used was required sieves to obtain $250-300 \mu \mathrm{m}$ (60-50 mesh) size which was obtained by sieve analysis. Representative particle sample were prepared, coated (sputtered) with platinum and examined under Scanning electron microscope. The abrasive particles were having irregular to angular shapes.

The slurry abrasion wear tests were performed using slurry abrasion test apparatus (Ducom make, India). A photograph of the Slurry abrasion tester is shown in Fig 3. The slurry abrasion tester is designed such that a flat test sample is pressed radially against a wheel with a known force. The test surface is submerged in wet abrasive media (slurry). The arrangement is such that the wheel carries the abrasive media between the sample and the wheel creating a situation of three body wear using the slurry in the chamber.

The specimens used for slurry abrasion testing were rectangular blocks measuring $57.2 \mathrm{~mm}$ (length) $\times 25.4 \mathrm{~mm}$ (width) $\times 9 \mathrm{~mm}$ (thickness). The specimens were polished using emery papers of $1 / 0$ and $2 / 0$ and cleaned with alcohol and dried with compressed air and then weighed using a digital electronic balance to the accuracy of $0.1 \mathrm{mg}$. The specimen was placed in the holder assembly. The desired load was placed on the loading lever and loading lever was released so that specimen comes in contact with rubber wheel. After the test was over the specimens were removed from the slurry chamber, cleaned with alcohol and weighed. The loss in mass (g) was calculated as the difference of initial and final weight of the specimen. In addition wear volume loss was also determined. In the present work slurry abrasion tests were carried out for martensitic stainless steel. The slurry abrasion tests were carried out to study the effect of load and slurry concentration on wear volume loss. The total numbers of revolutions were 2000, which was kept constant in all the tests.

\section{RESULTS AND DISCUSSION}

In the present work two different approaches were used to study slurry abrasive wear behavior of weld deposited martensitic stainless steel. The first approach aimed at measurement of material loss and second approach was based on visual and microscopic observations of worn out surfaces. Fig 4 shows then effect of sliding distance on wear volume loss of martensitic stainless steel at slurry concentration of $99.52 \%$ and load of 120 N. Fig. 5 shows the effect of slurry concentration on slurry abrasion volume loss as a function of normal load. Fig. 6 shows the effect of normal load on slurry abrasion volume loss as a function of slurry concentration. Figs. 7 ( $a$ and $b$ ) show photographs of typical worn out surfaces and Fig. 8 (a-c) show SEM photographs of worn out surfaces under different test conditions.

It can be observed that slurry abrasion volume loss increased linearly with increase in sliding distance as seen in Fig.4. With increasing slurry concentration, slurry abrasion volume loss increased significantly At 2000 revolution and at $120 \mathrm{~N}$ when the slurry concentration was increased from 27.07 to $54.14 \%$, the wear volume loss increased from 14.3 $\mathrm{mm}^{3}$ to $96.9 \mathrm{~mm}^{3}$, exhibiting nearly seven times increase for two fold increase in slurry concentration. Further increase in slurry concentration from 54.14 to $99.52 \%$ resulted in an increase in wear volume loss by more than three times as shown in Fig:5. At a load of $95 \mathrm{~N}$ the slurry abrasion volume loss exhibited twenty times increase, when slurry concentration was increased from 27.07 to $99.52 \%$ as shown in Fig 5 . At lower loads of $70 \mathrm{~N}$, an initial increase of more than five times in wear volume loss was observed, whereas further increase in slurry concentration from 54.14 to $99.52 \%$ resulted in more than 3.5 times increase in volume loss as shown in Fig. 5..At a load of $35 \mathrm{~N}$, the slurry abrasion volume loss exhibited an increasing trend with an increase in slurry 
concentration. However the magnitude of increase was fifteen times, which was less as compared to other test conditions.

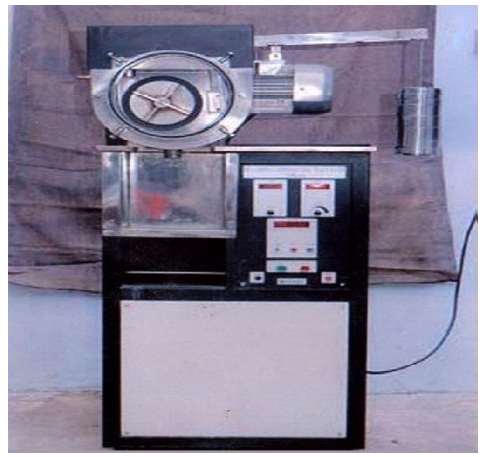

Fig 3 : Photograph of the slurry abrasion test apparatus

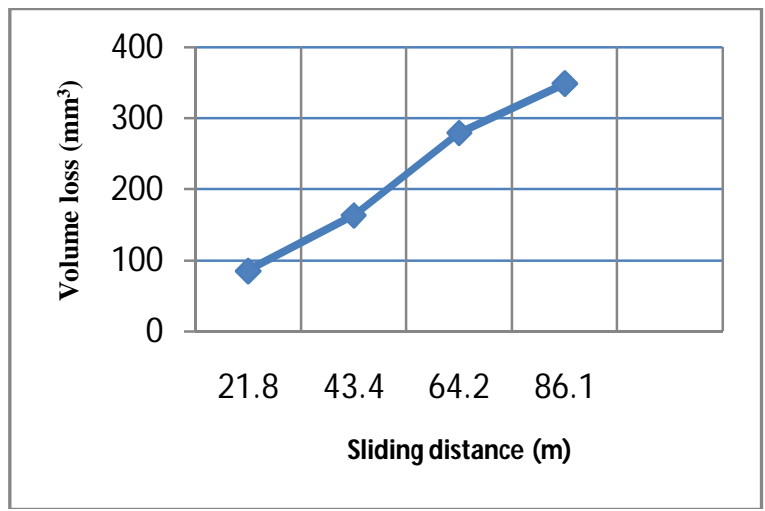

Fig 4 Effect of sliding distance on wear volume loss of martensitic stainless steel

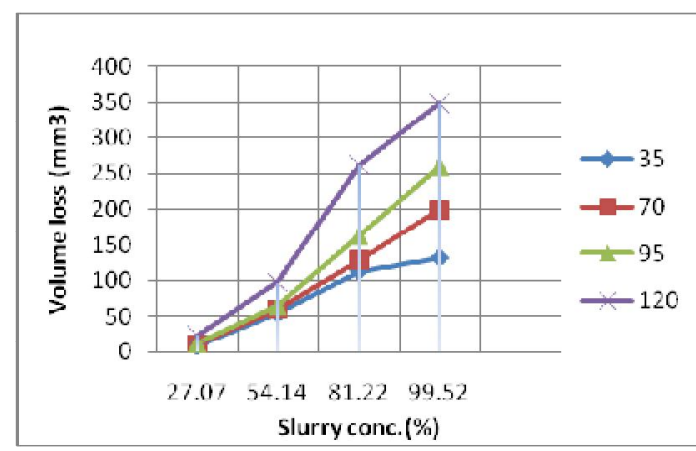

Fig 5 Slurry abrasion volume loss of weld deposited martensitic stainless steel vs slurry concentration as a function of normal load $(\mathrm{N})$ as shown in legends

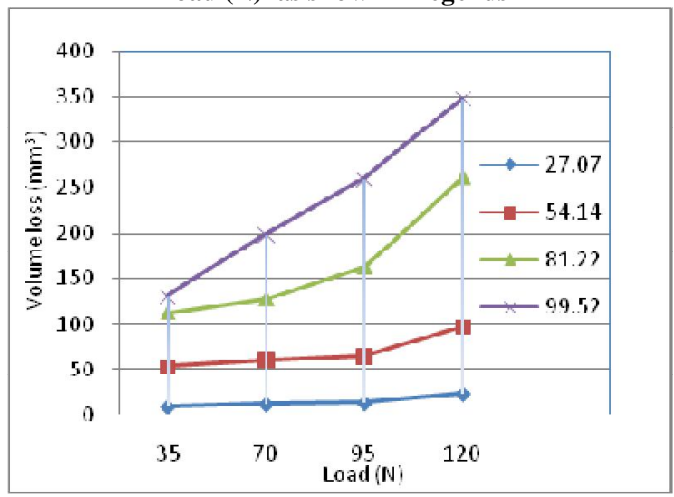

Fig 6 Slurry abrasion volume loss of weld deposited martensitic stainless steel vs slurry concentration as a function of slurry concentration $(\%)$ as shown in legends
It can be observed that slurry abrasion volume loss increased linearly with increase in sliding distance as seen in Fig.4. With increasing slurry concentration, slurry abrasion volume loss increased significantly.

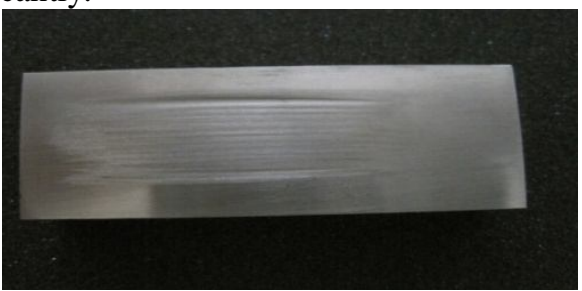

(a)

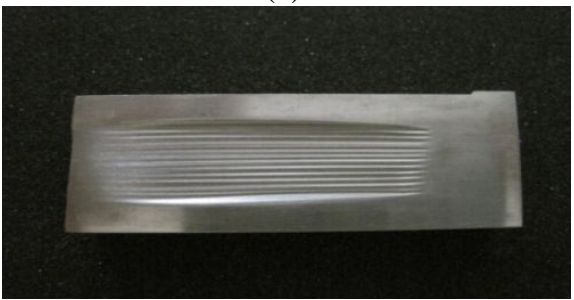

(b)

Fig. 7 (a and b) Photographs of worn out surfaces after slurry abrasion at a load of $120 \mathrm{~N}$ (a) at slurry concentration of 27.07 $\%$ and (b) at slurry concentration of $\mathbf{9 9 . 5 2 \%}$

At 2000 revolution and at $120 \mathrm{~N}$ when the slurry concentration was increased from 27.07 to $54.14 \%$, the wear volume loss increased from $14.3 \mathrm{~mm}^{3}$ to $96.9 \mathrm{~mm}^{3}$, exhibiting nearly seven times increase for two fold increase in slurry concentration. Further increase in slurry concentration from 54.14 to $99.52 \%$ resulted in an increase in wear volume loss by more than three times as shown in Fig:5. At a load of $95 \mathrm{~N}$ the slurry abrasion volume loss exhibited twenty times increase, when slurry concentration was increased from 27.07 to $99.52 \%$ as shown in Fig 5 . At lower loads of $70 \mathrm{~N}$, an initial increase of more than five times in wear volume loss was observed, whereas further increase in slurry concentration from 54.14 to $99.52 \%$ resulted in more than 3.5 times increase in volume loss as shown in Fig. 5..At a load of $35 \mathrm{~N}$, the slurry abrasion volume loss exhibited an increasing trend with an increase in slurry concentration. However the magnitude of increase was fifteen times, which was less as compared to other test conditions.

The effect of load on slurry abrasion volume loss is shown in Fig.6. for slurry concentration of 27.07, $54.14,81.22$ and $99.52 \%$ respectively. The slurry abrasion volume loss exhibited an increasing trend irrespective of the slurry concentration. However the magnitude of increase was different in each case. At lower concentration, the magnitude of increase was $1.4 \& 1.8$ times for slurry concentration of $27.07 \&$ $54.14 \%$ respectively. At higher slurry concentrations, the increase in wear loss was 2.2 to 2.7 times for slurry concentration of $81.22 \& 99.52 \%$ respectively. Thus it can be observed that at higher slurry concentration, the magnitude of increase in wear 
volume loss was nearly two times as compared to lower slurry concentration.

It is also observed from the results of the present work that wear volume loss obeys Archard's wear law;

$$
V=K S L / H
$$

Where $\mathrm{V}$ is volume loss, $\mathrm{K}$ is wear coefficient, $\mathrm{S}$ is sliding distance, $\mathrm{L}$ is normal load and $\mathrm{H}$ is the hardness of the surface (2). However at higher loads and slurry concentrations some deviations were observed. It was also observed that slurry concentration had relatively stronger influence on wear loss as compared to normal load [4,1314]. The photograph of worn out surfaces in Fig $7 \mathrm{a}$ and $b$ shows deep grooves and scratches on the surface at higher slurry concentration whereas at lower slurry concentration the surface is relatively smooth. At lower slurry concentration and lower load, silica particles are able to roll over the surface causing multiple number of indentation shown in Fig 8(a). The material is removed by ploughing mechanisms and lip of work hardened material can be seen attached to the grooves which resulted in lower slurry abrasion volume loss as compared to higher loads and higher slurry concentration. At higher loads of $70 \mathrm{~N}$ and slurry concentration of $54.14 \%$ worn out surface reveals continuous grooves caused by abrasive particles. The mechanism of material removal involves ploughing and cutting as shown in Fig 8(b). At higher loads of $120 \mathrm{~N}$ and slurry concentration of $99.52 \%$ the severely abraded surface can be seen in Fig 8(c). The predominant mechanisms involves micro cutting. With increasing load the depth and width of grooves is relatively greater causing more material loss and hence significant increase in slurry abrasion volume loss. It is also expected that with increasing load silica sand particle fractures generating additional fine particles. These fractured particles tend to have more angularity which also causes additional abrasion of the surface and contribute to increase in wear volume loss [15]. The above observations can explain significant increase in slurry abrasion volume loss with increase in normal load and slurry concentration which can also explain deviations from Archard's equation at higher loads and slurry concentrations

\section{CONCLUSIONS}

[1] The slurry abrasion volume loss of martensitic stainless steel increased with increase in slurry concentration and normal load.

[2] The magnitude of increase in slurry abrasion volume loss with respect to slurry concentration and normal load was different.

[3] The slurry concentration had more pronounced effect on slurry abrasion volume loss than normal load.
[4] The important mechanisms of material removal were ploughing, cutting and indentation. The predominant mechanism of material removal by abrasion was influenced by severity of test conditions.

[5] The slurry abrasion wear volume loss obeyed Arcahrd's wear law although some deviations were observed at higher loads and slurry concentrations which can be attributed to secondary abrasion caused by fragmented abrasive particles.

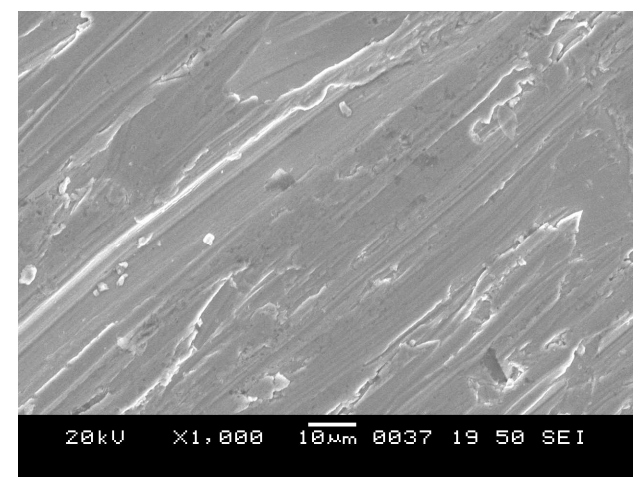

(a)

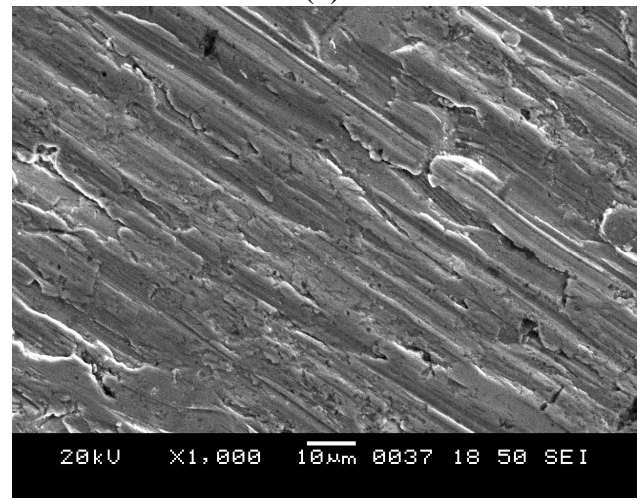

(b)

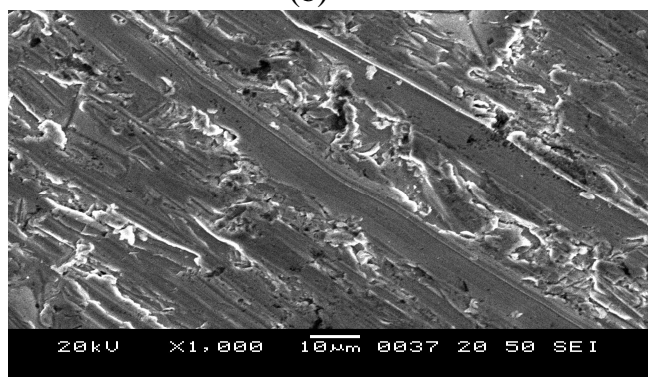

(c)

Fig. 8 (a-c) SEM photographs of worn out surfaces after slurry abrasion of martensitic stainless steel. (b) $70 \mathrm{~N}, 54.14 \%$ conc. (c) $120 \mathrm{~N}, 99.52 \%$ conc

\section{ACKNOWLEDGMENT}

The authors are thankful to Director VNIT for providing facilities for the present investigation. The authors also acknowledge the assistance of Ms Gauri in SEM work. 


\section{REFERENCES}

[1] G.W. Stachowiak, A.W. Batchelor, Engineering Tribology, Butterworth Heinemann

[2] I.M. Hutchings, Tribology Friction and Wear of Engineering Materials, Edward Arnold; 1992.

[3] P.J.Blau, Friction, Lubrication and Wear Technology. ASM Handbook.Vol.18.ASM International, The Materials Information Society, 1992.

[4] S.G. Sapate, A.D. Chopde, P.M. Nimbalkar, D.K Chandrakar, "Effect of microstructure on slurry abrasion response of En-31 steel," Mater \& Des, 29, pp 613-621, 2008

[5] Hozumi Goto, Yoshifumi, "Effect of varying load on wear resistance of carbon steel under unlubricated conditions,". Wear, 254, pp 1256-1266, 2003

[6] Ibrahim sevim, Eryurek IB, "Effect of abrasive particle size on wear resistance in steels," Mater \& Des, 27, pp 173-181, 2006

[7] G. Pintaude, F.G. Bernardes, "Mild and severe wear of steels and cast iron in sliding abrasion," Wear, 267, pp 1925,2009

[8] E. Badisch, C. Mitterer, "Abrasive wear of high speed steels,” Tribo Int, 36, pp 765-770, 2003
[9] Saghafian H, Kheirandish S, "Correlating microstructural features with wear esistance of dual phase steel," Mater Lett; 61, pp 3059-63, 2007.

[10] Adachi K, Hutchings I, "Sensitivity of wear rates in the micro-scale abrasion test to test conditions and material hardness". Wear , 258, pp :318-21,2005

[11] .Katsuki F, Watari K, Tahira H, Umino M, "Abrasive wear behavior of a pearlitic $(0.4 \% \mathrm{C})$ steel microalloyed with vanadium," Wear, pp 264, 331-336, 2008

[12] ZumGahr KH," Abrasive wear of two phase metallic materials with a coarse microstructure". In Ludema KC, ed. Proc of Int conf on wear of materials, Vancouver, ASME; April 1985. p. 45-57.

[13] Akira T, Takaoka T, Furukawa H, Hori H, Fukui T, Minami, "Development of abrasion resistant pipe for slurry transportation,” NKK Tech Rev,85, pp 16-22,2001

[14] Balasubramanian V, Varahamoorthy R, Ramachandran CS, Babu S, "Abrasive slurry wear behavior of stainless steel surface produced by plasma transferred arc hardfacing process," Surf Coat Technol , 202, pp 3903-12, 2008.

[15] G.P.Tilly and E.Sage, The interaction of particle and material behavior in erosion processes, Wear, 16, 447465,1970 . 